\title{
Planificación de la gestión de seguridad y salud en el trabajo en las universidades públicas del distrito de Riohacha, departamento de La Guajira, Colombia
}

\section{Planning of the Management of Safety and Health at work in the Public Universities of the District of Riohacha Department of La Guajira, Colombia}

Jaider Lopesierra-Orozco'; Esmider Alberto Serna-Ortiz²; Pedro Antonio Redondo-Silvera³

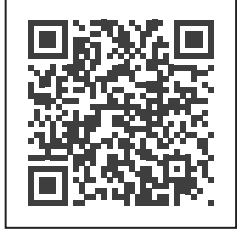

Palabras clave: salud ocupacional, riesgo laboral, seguridad en el trabajo, planificación, gestión, organización, dirección, universidad pública.

Articulo de investigación:

Fecha de recepción:

$16 / 05 / 2020$

Fecha de aceptación: $14 / 08 / 2020$

Esta publicación se encuentra bajo licencia:

Creative Commons

Reconocimiento-

NoComercial-

SinObraDerivada 4.0

Internacional

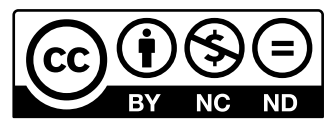

\section{Resumen}

El presente trabajo deriva de una investigación cuyo fin principal consistió en analizar la gestión de seguridad y salud en el trabajo en las universidades públicas del distrito de Riohacha en La Guajira, Colombia. Teóricamente se apoyó en autores del área de seguridad y salud en el trabajo, tales como la ley 1072 de 2015 (2015), Chiavenato (2007) y Münch y García (2008). En cuanto a su metodología, este trabajo fue de tipo descriptivo, no experimental, transeccional y de campo. Su población estuvo conformada por dos universidades del sector público, ubicadas en el distrito de Riohacha. Para recolectar la información, se acudió a un cuestionario conformado por 57 preguntas de escala tipo Likert. En relación con los resultados obtenidos, se concluyó que en lo atinente a la variable seguridad y salud

1 Administrador de empresas, especialista en gerencia en salud, magíster en Gerencia Empresarial. Universidad de La Guajira, Colombia. jjlopesierra@ uniguajira.edu.co, ORCID: https://orcid.org/0000-0002-1921-870X

2 Administrador de empresas, doctor en Ciencias Gerenciales, Universidad de La Guajira, Colombia. esmiderserna@uniguajira.edu.co, ORCID: https:// orcid.org/0000-0002-5922-5680

3 Contador público, abogado, magíster en Promoción y Protección de los Derechos Humanos, Universidad de La Guajira, Colombia. pa.redondo@ uniguajira.edu.co,ORCID: https://orcid.org/0000-0003-3229-7286 


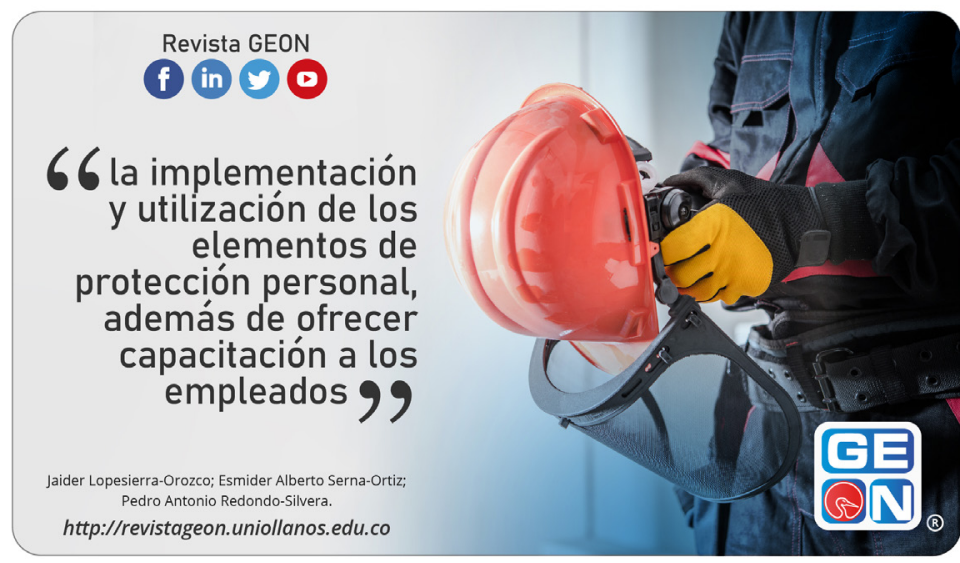

en el trabajo es necesario emplear herramientas administrativas de planificación, para consolidar los canales de comunicación establecidos dentro de la organización y del mismo modo incentivar al personal que labora con la finalidad de mejorar su desempeño. También se sugiere que impulsen de una manera exhaustiva la implementación y utilización de los elementos de protección personal, además de ofrecer capacitación a los empleados para que conozcan los objetivos que se desean alcanzar, específicamente, los relacionados con la variable objeto de estudio. Asimismo, se propone crear e implementar programas de seguridad y salud en el trabajo que den respuestas a estas necesidades.

Palabras clave: salud ocupacional, riesgo laboral, seguridad en el trabajo, planificación, gestión, organización, dirección, universidad pública.

Códigos JEL: J2, J24, J28, J36, J8, J81, J83, M1, M11, M12, M54

\section{Abstract}

Cómo citar este artículo /

Toreference this article:

Lopesierra Orozco, J., Serna Ortiz, E. A., \& Redondo Silvera, P. A. (2020). Planificación de la Gestión de Seguridad y Salud en el trabajo en las Universidades Públicas del Distrito de Riohacha Departamento de La Guajira, Colombia. Revista GEON (Gestión, Organizaciones Y Negocios), 7(2), 1-13. https://doi. org/10.22579/23463910.214

The present work derives from an investigation whose main purpose was to analyze the management of safety and health at work in the public universities of the district of Riohacha in La Guajira, Colombia. Theoretically, it was based on authors from the area of safety and health at work, such as, law 1072 of 2015 (2015), Chiavenato (2007), and Münchand García(2008). Regardingitsmethodology, 
Cómo citar este artículo /

Toreference this article:

Lopesierra Orozco, J., Serna Ortiz, E. A., \& Redondo Silvera, P. A. (2020). Planificación de la Gestión de Seguridad y Salud en el trabajo en las Universidades Públicas del Distrito de Riohacha Departamento de La Guajira, Colombia. Revista GEON (Gestión, Organizaciones Y Negocios), 7(2), 1-13. https://doi. org/10.22579/23463910.214 this work was descriptive, non-experimental, cross-sectional, and field-based. Its population was made up of 2 universities in the public sector, located in the district of Riohacha. To collect the information, a questionnaire consisting of 57 Likert-type scale questions was used. In relation to the results obtained, it was concluded that regarding the variable occupational health and safety, it is necessary to use administrative planning tools to consolidate the communication channels established within the organization, in the same way, to encourage staff that works in order to improve its performance. It is also suggested that they exhaustively promote the implementation and use of personal protection elements, in addition to offering training to employees so that they know the objectives that they wish to achieve, specifically, those related to the variable under study. Likewise, it is proposed to create and implement occupational safety and health programs that respond to these needs.

Keywords: Occupational health; Occupational risk; Safety at work; Planning; Management; Organization; Direction; Public university.

\section{Introducción}

El objetivo de este trabajo consistió en analizar la planificación de la seguridad y salud en el trabajo en las universidades públicas del distrito de Riohacha, departamento de La Guajira, Colombia, teniendo en cuenta la importancia que ha ganado esta temática a nivel de organismos supranacionales, como la Organización de las Naciones Unidas y la Organización Internacional del Trabajo, de los mismos estados, del sector empresarial y, desde luego, de los trabajadores, para quienes significa una conquista.
Sin embargo, según datos de la Federación de Aseguradores Colombianos ([Facecolda], 2018), citados por el Consejo Colombiano de Seguridad (2019), el número de enfermedades calificadas durante el 2018 fue de 10.435 casos, presentándose así un aumento del $7,1 \%$ con respecto al 2017; en cuanto a la mortalidad, se presentaron 569 muertes de origen laboral. Esto es un indicativo de que, a pesar de las medidas adoptadas por el Estado, los resultados no han sido los esperados. 
Finalmente, en lo tocante a la estructura del artículo, tenemos que, inicialmente, se presentan las bases teóricas que sustentan la variable objeto de estudio, la dimensión y sus indicadores. Posteriormente, se describe la arquitectura metodológica que se adoptó para poder conseguir los objetivos propuestos. Por último, se aborda el análisis y discusión de los resultados que soportaron las conclusiones y las recomendaciones.

\section{Contexto teórico}

Para poder abordar el contexto teórico, es indispensable mencionar que el decreto 1072 de 2015, en su capítulo 6 , describe los lineamientos que de manera obligatoria deben tener en cuenta tanto los empleadores públicos como los privados para poder implementar el sistema de gestión de seguridad y salud en el trabajo (SGSST). De igual manera, debemos traer a colación la ley 1562 de 2012, que establece una serie de lineamientos y definiciones básicas relacionadas con el sistema de riesgos laborales.

En esta misma dirección, Chiavenato (2007) refiere que "la seguridad laboral es el conjunto de medidas técnicas, educativas, médicas y psicológicas utilizadas para prevenir accidentes" (p. 336). En este sentido, de acuerdo con Münch y García (2008), la seguridad laboral está conformada por varias fases: planificación, organización, dirección y control.

De igual manera, es importante citar la resolución 0312 de 2019, expedida por el Ministerio de Trabajo (2019), "Por la cual se definen los estándares mínimos del sistema de gestión de la seguridad y salud en el trabajo". Así las cosas, es una obligación de tipo legal implementar el SG-SST y gestionar la seguridad y salud ocupacional. De acuerdo con Álvarez, Navarro, Montoya y Gil (2018, p. 41), esta gestión es una herramienta de la administración que permite dirigir las acciones que se desean implementar en la organización para que esta pueda ser enfocada hacia toda la organización o hacia un área específica, con lo que se busca generar un desarrollo óptimo de los procesos y cumplir con los objetivos propuestos.

En este punto, es pertinente mencionar que las instituciones objeto de estudio cuentan con una dependencia única para seguridad y salud en el trabajo, la cual está adscrita a la dirección de talento humano, desde la cual se coordinan las acciones que permiten adoptar, implementar y ejecutar todos los procesos conducentes a mitigar y prevenir accidentes y enfermedades de tipo laboral, a través de la vigilancia, el seguimiento y el control en los procesos.

En el mismo orden de ideas, según Lema-Cruz y Álvarez-Lema (2016), "la planeación busca establecer soluciones a los problemas y aplica métodos de acuerdo a la experiencia que tiene el estratega" (p. 167). Por su parte, Chiavenato (2007) expresa que la planeación es la función administrativa que determina anticipadamente cuáles son los objetivos que deben alcanzarse y qué debe hacerse para alcanzarlos. Münch y García (2008) sostienen que la planeación "[...] in- 
volucra hacer la elección de las decisiones más adecuadas acerca de lo que se va a hacer en el futuro" (p. 41). Finalmente, Robbins y Coulter (2010) afirman que "la planificación implica definir los objetivos de la organización, establecer estrategias para lograr dichos objetivos y desarrollar planes para integrar y coordinar actividades de trabajo" (p. 144).

En lo atinente a los propósitos, Vilariño (2017) expresa que, desde la óptica de las empresas, el propósito viene a ser el motivo por el cual estas existen y que trasciende el deseo de generar dinero. En relación con los objetivos, Taype (2015) afirma que son declaraciones que identifican el punto final o condición que desea alcanzar una organización. De igual manera, Serna (2010) indica que "los objetivos son los resultados globales que una organización espera alcanzar en el desarrollo y operacionalización concreta de su misión y visión" (p. 225).

De otra parte, Para Ramos (2008) "la estrategia es una posición cuando se ve como un medio de situar una organización en lo que los teóricos de la organización denominan como entorno" (p. 11). Finalmente, Münch y García (2008, p. 40) expresan que las estrategias son cursos de acción general o alternativas que muestran la dirección y el empleo de los recursos y esfuerzos, a fin de prevenir o controlar los accidentes y enfermedades ocupacionales en las condiciones más ventajosas.

En relación con las políticas, para la normativa internacional OHSAS
18001 (2007), la política es definida como la intensión y dirección general de una organización relacionada con su desempeño formalmente expresado por la alta dirección; esta proporciona una estructura para la acción y el establecimiento de los objetivos. Koontz y Weihrich (2013) explican "que las políticas son planes, declaraciones o ideas generales que guían el proceso de toma de decisiones y aseguran que sea congruente y contribuya al logro de los objetivos" (p. 80).

En cuanto al presupuesto, Correa (2011), citado por Parra y La Madrid (2017), "expresa que el presupuesto es una herramienta administrativa de planeación y control financiero donde se presentan ordenadamente y en términos monetarios los resultados previstos de un plan, un proyecto, una estrategia" (p. 37). Finalmente, Burbano (2011) manifiesta que "el presupuesto surge como herramienta moderna de planeación y control al reflejar el comportamiento de la competencia y de indicadores económicos y su relación con los diferentes aspectos administrativos, contables y financieros de la empresa" (p. 11).

\section{Materiales y métodos}

Esta investigación se desarrolló bajo el enfoque cuantitativo. Según Cabezas, Andrade y Torres (2018), este enfoque "se fundamenta y utiliza la observación del proceso en forma de recolección de datos y los analiza para llegar a responder las preguntas que se plantean en un inicio de la investigación" (p. 66). Asimismo, este estudio se caracterizó por ser descriptivo, 
ya que, de acuerdo con Ríos-Ramirez (2017), "busca encontrar las características, comportamientos y propiedades del objeto de estudio" (pág. 89).

Por otra parte, en lo relacionado con el diseño, se concibe como una investigación de campo debido a que el investigador obtiene la información sin alterarla; solo la alteran las condiciones existentes (Arias, 2016); de ahí su carácter de investigación no experimental. Finalmente, el estudio es de carácter transversal, tal y como lo refiere Ríos-Ramírez, (2017), porque "realiza la recolección de datos en un corto período o un determinado punto del tiempo" (p. 93).

Para determinar la población del presente estudio, se emplearon de manera intencional criterios de selección previamente definidos por el investigador, tales como: instituciones del sector universitario público del distrito de Riohacha, que posean en su estructura organizativa formalmente establecido un departamento de seguridad y salud en el trabajo. Así las cosas, su población estuvo constituida por 22 trabajadores del nivel administrativo-directivo, de los cuales, 6 intervienen en la etapa de planificación, y 76 trabajadores administrativos-operativo, de los cuales, 12 actúan en la etapa operativa. Finalmente, esta investigación se abordó como censo poblacional

En relación con el tipo de información, tenemos que "cuando se recorren todas las etapas del momento de la producción de los datos, significa que en esa investigación se está traba- jando con fuentes primarias" (Cohen, 2019, p. 256). En este sentido, las fuentes primarias de esta investigación están relacionadas con la información suministrada por la población objeto de estudio, información que se recolectó mediante la aplicación de un instrumento. Al respecto, Arias (2016) establece que el instrumento de recolección de datos es todo aquel medio, mecanismo o representación en documento o digital que se utiliza para obtener una recopilación de información. Este instrumento consistió en un cuestionario de preguntas cerradas que dan cuenta de la variable gestión de seguridad y salud en el trabajo y 3 dimensiones. En este caso particular, se abordó la correspondiente a la planificación de la gestión de seguridad y salud en el trabajo mediante 21 ítems diseñados para dar cuenta de la existencia, aplicación y eficacia de los objetivos, estrategias, políticas, programas, presupuesto y procedimiento que constituyen los indicadores de la variable.

Por otra parte, se tiene que la información se abordó utilizando la herramienta de Baremo, estableciendo los siguientes intervalos: de 1,00 a 1,80 se considera como ausente; de 1,81 a 2,60, muy baja presencia; de 2,61 a 3,40 , baja presencia; de 3,41 a 4,20, medianamente presente; y de 4,21 a 5,00 , presente. Finalmente, la validez del instrumento se obtuvo por parte de cinco expertos, quienes determinaron la pertinencia y correspondencia de la variable, así como también con las dimensiones y los indicadores establecidos. A fin de estimar la confiabilidad del instrumento, se procedió a aplicar una prueba piloto en la 
que se utilizó el método coeficiente Alfa Cronbach y se obtuvo un valor de 0,96, lo que nos indica un resultando con una alta confiabilidad. Yuni y Urbano (2014) definen confiabilidad como "la capacidad del instrumento para arrojar datos o mediciones que correspondan a la realidad que se pretende conocer" (p. 33).

Dado lo anterior, para la sociedad, la pertinencia del estudio representa un referente que debe ser implantado en el área de la seguridad y salud en el trabajo vista desde la óptica gerencial y para futuros estudios, ya que podrá ofrecer información científica que permitirá conducir a las empresas e investigadores a estar atentos en el cumplimiento de las normas, planes y programas en las políticas de bienestar laboral y social.

\section{Análisis de los resultados}

El análisis de los resultados se representa mediante tablas donde se detallan las frecuencias absolutas y relativas de cada ítem, así como el promedio de estas. Dichos resultados permitieron conocer de manera amplia las debilidades y fortalezas de la ejecución de la gestión de seguridad y salud en el trabajo.

La tabla 1 muestra el comportamiento de las respuestas en función de la dimensión planeación de la seguridad y salud en el trabajo. En relación con el indicador Propósitos, se observa que un 37,50\% de los encuestados eligió la alternativa Casi siempre como respuesta, mientras que un 33,33\% optó por la opción $A$ veces, con lo que se obtuvo una media de 3,10, que, comparada con el Baremo, muestra una baja presencia; es decir, que dentro de las universidades, los propósitos se encuentran poco establecidos y definidos en relación con el logro de los objetivos y la identificación de estas en cuanto a la planificación de la gestión de seguridad y la salud en el trabajo.

Tabla 1. Dimensión: planeación administrativa

\begin{tabular}{lc|c|c|c|c|c|c|c|c|c|c}
\multirow{2}{*}{ Indicaciones } & \multicolumn{2}{c|}{ Siempre } & \multicolumn{2}{c|}{ Casi siempre } & \multicolumn{2}{c|}{ A veces } & \multicolumn{2}{c|}{ Casi nunca } & \multicolumn{2}{c|}{ Nunca } & \multirow{2}{*}{ Media } \\
\cline { 2 - 13 } & FA & FR & FA & FR & FA & FR & FA & FR & FA & FR & \\
\hline Propósitos & 1,58 & 2,08 & 28,5 & 37,5 & 25,33 & 33,33 & 17,41 & 22,92 & 3,16 & 4,17 & 3,10 \\
\hline Objetivos & 0,00 & 0,00 & 41,16 & 54,17 & 28,5 & 37,5 & 4,75 & 6,25 & 1,58 & 2,08 & 3,44 \\
\hline Estrategias & 1,58 & 2,08 & 36,41 & 47,92 & 31,66 & 41,67 & 6,33 & 8,33 & 0,00 & 0,00 & 3,44 \\
\hline Políticas & 0,00 & 0,00 & 31,66 & 41,67 & 28,5 & 37,5 & 15,83 & 20,83 & 0,00 & 0,00 & 3,21 \\
\hline Programas & 0,00 & 0,00 & 20,5 & 27,08 & 25,33 & 33,33 & 30,08 & 39,58 & 0,00 & 0,00 & 2,88 \\
\hline Presupuestos & 13,00 & 81,25 & 2,67 & 16,67 & 0,00 & 0,00 & 0,33 & 2,08 & 0,00 & 0,00 & 4,77 \\
\hline Procedimientos & 3,16 & 4,17 & 25,33 & 33,33 & 23,75 & 31,25 & 23,75 & 31,25 & 0,00 & 0,00 & 3,1 \\
Media & $\mathbf{2 , 7 6}$ & $\mathbf{1 2 , 8}$ & $\mathbf{2 6 , 6}$ & $\mathbf{3 6 , 9}$ & $\mathbf{2 3 , 2 9}$ & $\mathbf{3 0 , 7}$ & $\mathbf{1 4 , 0 6}$ & $\mathbf{1 9}$ & $\mathbf{0 , 6 7}$ & $\mathbf{0 , 8 9}$ & $\mathbf{3 , 4 2}$ \\
\hline
\end{tabular}

Fuente: elaboración propia. 
En cuanto al indicador Objetivos, un $54,17 \%$ de la población encuestada se inclinó por la opción Casi siempre, mientras que un 37,50\% escogió como opción la alternativa $A$ veces, 6,25\% escogió la opción Casi nunca y 2,08\% la opción Nunca en lo relacionado con el alcance de los objetivos en el futuro y las acciones realizadas, donde se obtuvo una media de 3,44, la cual, comparada con el Baremo, señala un mediano cumplimiento; es decir, los objetivos sirven a medias como guía en la realización de actividades o tareas para alcanzar las metas propuestas.

En relación con el indicador Estrategias, el 47,92\% de los encuestados eligió la opción de respuesta Casi siempre en lo que respecta a la formulación de estrategias acordes para alcanzar el logro de los objetivos planteados, mientras que un $41,67 \%$ escogió como alternativa de respuesta la opción $A$ veces, un $8,33 \%$ se inclinó por la opción Nunca y un 2,08\% escogió Siempre, lo que alcanzó una media de 3,44. Esto se puede calificar como medio en comparación con el Baremo aplicado. Se observa que en las universidades objeto de estudio no se determinan totalmente los objetivos básicos a largo plazo y la adopción de líneas de acción, así como la asignación de los recursos necesarios para la consecución de dichas metas.

Con respecto al indicador Políticas, se observa que la alternativa de respuesta con mayor porcentaje corresponde a Casi siempre, con un 41,67\%, mientras que un 37,50\% respondió a favor de la opción $A$ veces y un $20,83 \%$ a favor de Casi nunca. Se obtuvo así una media de 3,21, lo que lo califica como baja presencia dentro del Baremo establecido. Esto indica que, dentro de las universidades objeto de estudio, las políticas establecidas no cumplen por completo la función en relación con la toma de decisiones y la regulación del comportamiento del personal con base en las medidas que deben implementarse al desarrollar su labor.

Por su parte, en lo que respecta al indicador Programas, se evidencia que un 39,58\% de la población eligió la posibilidad de Casi nunca, un 33,33\% señaló Nunca y un 27,08\% optó por Casi siempre, con lo que se obtuvo una media de 2,88, lo cual, comparado con el Baremo planteado, muestra una baja presencia. Lo anterior afirma que las universidades objeto de estudio no siempre establecen programas que reflejan la secuencia de actividades y el tiempo establecido para el logro de estas.

En cuanto el indicador Presupuesto, un $81,25 \%$ de la población encuestada se inclinó por la opción de respuesta Siempre, un 16,67\% seleccionó Casi siempre y un 2,08\% eligió Casi nunca, con lo que se obtuvo una media de 4,77, lo cual lo califica como alta presencia dentro del Baremo planteado. En consecuencia, se afirma que dentro de las universidades se realizan presupuestos en términos monetarios acordes con los desembolsos a realizar en un tiempo determinado vinculados a la planificación de la gestión de seguridad y salud en el trabajo. 
Por último, está el indicador Procedimientos, donde se evidencia que un 33,33\% de la población eligió la posibilidad de respuesta Casi siempre, mientras que un 31,25\% de la población escogió la opción $A$ veces, un 31,25\% la opción Casi nunca y un $4,17 \%$ la opción Siempre, con lo que se obtuvo una media de 3,10, lo cual, comparado con el Baremo, refleja una baja presencia. Lo anterior muestra que, dentro de las universidades objeto de estudio, no se llevan medios de normalización de todas las operaciones y actividades que se realizan integrando en estos los aspectos de seguridad y salud en el trabajo que sean precisos para controlar los riesgos derivados de tales actividades.

\section{Discusión de los resultados}

Luego de analizar los datos recopilados, se estableció que los Propósitos se encuentran poco establecidos y definidos en relación con la consecución de los objetivos. Lo anterior discrepa de lo manifestado por Münch y García (2008), que refieren que "los propósitos son las aspiraciones cualitativas básicas en el orden moral que mueve a emprender acciones de tipo socioeconómico y que se establecen en forma permanente o semipermanente en un grupo social" (p. 242).

En lo que corresponde a los Objetivos, se observa que estos guían a medias la realización de actividades tendientes a lograr la gestión de seguridad y salud en el trabajo en las instituciones bajo estudio. Así las cosas, es evidente la discrepancia con lo planteado por Münch y García (2008), quienes manifiestan "que los objetivos representan los resultados que la empresa espera obtener; son fines por alcanzar, establecidos cuantitativamente y determinados para realizarse transcurrido un tiempo específico" (p. 96).

En lo atinente a las Estrategias, se estableció que en estas instituciones no se determinan totalmente los objetivos básicos a largo plazo, ni se asignan los recursos suficientes para alcanzarlos. Esto contrasta con los planteamientos de Münch y García (2008), quienes explican que "las estrategias son cursos de acción general o alternativas, que muestran la dirección y el empleo de los recursos y esfuerzos, a fin de prevenir o controlar los accidentes y enfermedades laborales en las condiciones más ventajosas" (p. 40).

A su vez, las políticas establecidas no cumplen a cabalidad su función relacionada con la toma de decisiones y la regulación del comportamiento del personal. Estos resultados divergen con lo expuesto por Münch y García (2008), que definen las políticas como "disposiciones del pensamiento administrativo que orientan o regulan la conducta que hay que seguir en la toma de decisiones, acerca de acciones o actividades que se repiten una y otra vez dentro de una organización" (p. 86).

Por otra parte, se tiene que estas instituciones no siempre establecen programas que indiquen la secuencia de actividades y el tiempo establecido para la consecución de las metas. Esta situación discrepa de lo planteado 
por Münch y García (2008), debido a que expresan que los programas "son un esquema en donde se establece la secuencia de actividades específicas que deberán realizarse para alcanzar los objetivos, y el tiempo requerido para efectuar cada una de sus partes y todos aquellos eventos involucrados en su consecución" (p. 182).

En lo correspondiente al Presupuesto, tenemos que las universidades objeto de estudio realizan presupuestos ajustados a los niveles de recursos que esperan desembolsar en un tiempo determinado, vinculados a la planificación y salud en el trabajo. Esta situación concuerda con lo expresado por Münch y García (2008), debido a que plantean que el presupuesto "es un esquema escrito de tipo general y específico, que determina por anticipado, en términos cuantitativos (monetarios y no monetarios) el origen y asignación de los recursos de la empresa, para un periodo específico" (p. 187).

Finalmente, en torno a los Procedimientos, se tiene que al interior de estas instituciones no se aplican medios que tiendan a la normalización de todas las operaciones y actividades que se realizan integrando en ellos los aspectos de seguridad y salud en el trabajo que sean necesarios para controlar los riesgos derivados de tales actividades.

En resumen, al analizar conjuntamente las respuestas que se obtuvieron para cada uno de los indicadores que conforman la planeación administrativa, se evidencia que la dimensión tuvo como resultado una media de
3,42, considerada media de acuerdo con el Baremo establecido en la investigación. Lo anterior significa que, dentro de las universidades objeto de estudio, los sistemas que conforman la planificación se encuentran medianamente establecidos en lo que respecta a la gestión de seguridad y la salud en el trabajo. También discrepa de los planteamientos de Münch y García (2008, p. 41), quienes expresan que la planeación antecede a las demás etapas que conforman el proceso administrativo, pues planear involucra hacer la elección de las decisiones más adecuadas acerca de lo que se va a realizar en el futuro. Asimismo, afirman que la planeación establece las bases para determinar el elemento riesgo y minimizarlo.

Actualmente, la preocupación por parte de los estados y de las instituciones públicas o privadas por tratar de garantizar las condiciones laborales necesarias para preservar la salud física y mental de sus trabajadores se ha acentuado notablemente debido a su obligación de ponerse a tono con los diferentes escenarios que, en materia laboral, le ofrece este mundo moderno. Así las cosas, la seguridad y salud de los trabajadores pertenecientes a las universidades no escapa a la situación menciona$\mathrm{da}$, donde estos lineamientos buscan dar respuesta a las necesidades atinentes a la gestión de seguridad y salud en el trabajo. En ese sentido, Dressler (2015) refiere que la capacitación en seguridad es otra forma de reducir las conductas inseguras, en especial con los trabajadores nuevos. Es necesario instruirlos en 
prácticas y procedimientos de seguridad, advertirles acerca de los peligros potenciales y trabajar para crear una actitud de conciencia para la seguridad. Las normas de la OSHA determinan que la capacitación no es suficiente. El personal debe demostrar que realmente sabe lo que hay que hacer (p. 438).

La formulación de lineamientos para la optimización de la gestión de seguridad y salud en el trabajo en las universidades objeto de estudio permitirá un proceso integrado en cuanto a la seguridad y salud de los trabajadores, dado que estos procedimientos vinculados a la actividad laboral deben brindar las condiciones seguras para ejercer las funciones asignadas, propiciar las condiciones que permitan adoptar el programa de seguridad y salud, ajustado a las normas existentes para tal fin, y así dar cumplimiento a los requisitos exigidos a las instituciones.

De acuerdo con los resultados, las universidades deben desarrollar procedimientos para la planificación de la gestión de seguridad y salud en el trabajo mediante estrategias de acción que den a conocer las políticas de seguridad y salud en el trabajo y la promoción de la comprensión de las medidas adoptadas en todos los niveles de la organización, a través de la planificación, organización, dirección y desarrollo del sistema de gestión de seguridad y salud en el trabajo y de la invitación de todo el personal a participar en las actividades tendientes a diseñar los planes de acción. De la misma manera, se deben desarrollar procedimientos y acciones que garanticen los resultados deseados por la organización mediante labores de coordinación con los jefes de áreas para la evaluación y actualización de la matriz y la identificación de peligros evaluando y valorando los riesgos y priorizando su intervención.

\section{Conclusiones}

Los resultados obtenidos en la presente investigación hicieron posible el análisis de la planificación de la seguridad y salud en el trabajo en las universidades públicas del distrito de Riohacha, en el departamento de La Guajira, Colombia. En consecuencia, una vez recolectada y analizada la información se pudo llegar a las siguientes conclusiones:

- En cuanto a este fin, con el cual se busca describir la planeación de la gestión de seguridad y salud en trabajo en las universidades públicas del distrito especial turístico y cultural de Riohacha, en el departamento de La Guajira, Colombia, Ios propósitos se encuentran medianamente establecidos y definidos en relación con el logro de los objetivos y la identificación de estos.

- A su vez, no se adoptan totalmente las líneas de acción ni se asignan los recursos que se necesitan para poder alcanzar los objetivos.

- De igual manera, las políticas establecidas no cumplen por completo su función en cuanto a la toma de decisiones y la regulación del comportamiento del personal. 
- Por último, no siempre establecen programas que reflejan la secuencia de actividades y el tiempo establecido para el logro de estas en cuanto a la planificación de la gestión de seguridad y la salud en el trabajo.

Teniendo en cuenta las situaciones anteriormente descritas, se puede afirmar que existen limitaciones en cuanto a la correcta aplicación de los propósitos, estrategias, objetivos, políticas y procedimientos que pertenecen a la fase de planeación. Así las cosas, se sugiere que impulsen de una manera exhaustiva cada uno de los elementos preventivos de seguridad que la conforman, con la finalidad de que los miembros de la organización puedan conocer un poco más sobre los objetivos que se desean alcanzar. Se propone, entonces: crear e implementar programas que capaciten a los empleados con el fin de que estos puedan identificar los peligros y controlar los riesgos de su entorno laboral, que den respuestas a estas necesidades; formular lineamientos desde las autoridades universitarias; implementar un programa de acciones acorde con las necesidades específicas detectadas en la identificación de peligros, evaluación y valoración de riesgos; y participar en la prevención de riesgos laborales para satisfacer las necesidades en cuanto a seguridad y salud en el trabajo en las universidades objeto de estudio.

\section{Referencias}

Álvarez, L. A., Navarro, D., Montoya, A. V., \& Gil, A. (2018). Modelos de gestión organizacional y planeación es- tratégica de las clínicas odontológicas. Revista Gestión, Organizaciones y Negocios, 5(2), 38-49. https://doi. org/10.22579/23463910.32

Arias, F. (2016). El proyecto de investigación, introducción a la metodología científica (7. ${ }^{\text {a ed.). Episteme. }}$

Behar, S. (2008). Introducción a la metodología de investigación. Shalom.

Burbano, J. (2011). Presupuesto, enfoque de gestión, planeación y control de recursos (4. ${ }^{a}$ ed.). McGraw-Hill S.A.

Cabezas, E., Andrade, D., \& Torres, J. (2018). Introducción a la metodología de la investigación científica. Universidad de las Fuerzas Armadas ESPE. https://bit.ly/3lxVgkk

Chiavenato, I. (2007). Administración: proceso administrativo (3. ${ }^{a}$ ed.). McGraw-Hill.

Cohen, N. (2019). Metodología de la investigación, ¿para que?: la producción de los datos y diseños. Teseo.

Cómo le fue a Colombia en accidentalidad, enfermedad y muerte laboral en 2018. (2019). Consejo Colombiano de Seguridad. https://bit.ly/2QCTMXX

Dressler, G. (2015). Administración de recursos humanos (14. ${ }^{\text {a }}$ ed.). Pearson.

Flores, M. H., \& Perdomo, C. A. (2015). El conocimiento estratégico y gerencial de competitividad para las microempresas en Colombia. Global Conference on Business and Funance Proceedings, 10(2), 1282-95.

Grupo de Proyecto OHSAS. (2007). OHSAS 18001:2007. Sistemas de gestión de la seguridad y salud en el trabajo. AENOR Internacional.

Koontz, H. \& Weihrich, H. (2013). Elementos de administracion: Un enfoque in- 
ternacional (7.a ed.). McGraw-Hill Interamericana.

Lema-Cruz, J., \& Álvarez-Lema, F. (2016). Factores determinantes en la planeación estratégica. Revista UTCiencia, 3(33), 166-80. https://bit.ly/31xxSDr

Münch, L., \& García, J. (2008). Fundamentos de administración (10. a ed.). Trillas.

Parra, J., \& La Madrid, J. (2017). Presupuesto como instrumento de control financiero en pequeñas empresas de estructura familiar. Revista Negotium, 13(38), 33-48. https://www.redalyc. org/pdf/782/78253678003.pdf

Presidencia de la República. (2012, 11 de julio). Ley 1562. Por la cual se modifica el sistema de riesgos laborales y se dictan otras disposiciones en materia de salud ocupacional. Diario Oficial 48488. https://bit.ly/2QzHjEa

Presidencia de la República. (2019, 13 de febrero). Resolución 0312. Por la cual se definen los estándares mínimos del sistema de gestión de la seguridad y salud en el trabajo SG-SST. Diario Oficial 50872. https://bit.ly/3hlxob3

Presidencia de la República. (2020, 26 de mayo). Decreto 1072. Por medio del cual se expide el decreto único reglamentario del sector trabajo. Diario Oficial 49523. https://bit.ly/3bbq8SX
Ramos, A. (2008). Cómo llegan a la definición de la estrategia las empresas localizadas en el Valle de Aburrá. Revista Escuela de Ingeniería de Antioquia, 9, 9-29. https://www.redalyc.org/ pdf/1492/149216913001.pdf

Ríos-Ramírez, R. (2017). Metodología para la investigación y redacción. Servicios Académicos Intercontinentales S.L. $\quad$ http://www.eumed.net/libros/libro.php?id=1662

Robbins, S. \& Coulter, M. (2010). Administración (10. ${ }^{a}$ ed.). Editorial Pearson Educación.

Serna, A. (2010). Gerencia estratégica

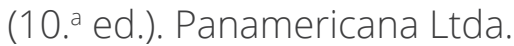

Taype, M. (2015, 9 de noviembre). Objetivos organizacionales. Gestiopolis. www.gestiopolis.com/objetivos-organizacionales/

Vilariño, A. (2017, 28 de agosto). El propósito en las organizaciones, clave en la relación empresa-trabajador. Compromiso Empresaria. https://bit. ly/2QEtq70

Yuni, J., \& Urbano, C. (2014). Técnicas para investigar: recursos metodológicos para la preparación de proyectos de investigación (2. ${ }^{a}$ ed.). Brujas. 